\title{
Pricing Without Equivalent Martingale Measures Under Complete and Incomplete Observation
}

\author{
Wolfgang J.Runggaldier and Giorgia Galesso
}

\begin{abstract}
Traditional arbitrage pricing theory is based on martingale measures. Recent studies show that there are situations when there does not exist an equivalent martingale measure and so the question arises: what can one do with pricing and hedging in this situation? We mention here two approaches to this effect that have appeared in the literature, namely the "Fernholz-Karatzas" approach and Platen's "Benchmark approach" and discuss their relationships both in models where all relevant quantities are fully observable as well as in models where this is not the case and, furthermore, not all observables are also investment instruments.
\end{abstract}

\section{Introduction}

Traditional arbitrage pricing theory is based on martingale measures, whereby the density process as well as the prices expressed in units of a same numeraire are martingales. Recent studies such as [1],[2] show that some form of arbitrage may exist in real markets and then processes, which in traditional theory are martingales, turn out to be strictly local martingales. Without necessarily passing through absence of arbitrage, also the authors of [5] describe instances in which classical martingale processes are strict local martingales. Typically such a process is the density process and then no equivalent martingale measure exists. In such a situation the question arises: what can one do with pricing and hedging?

This issue has already been dealt with in the literature and, after recalling the basic features of two of these approaches, our first purpose in the paper is to show that,

Wolfgang J.Runggaldier

Department of Pure and Applied Mathematics, University of Padova, 63,Via Trieste, I-35121

Padova, Italy e-mail: runggal@ math.unipd.it

Giorgia Galesso

Department of Pure and Applied Mathematics, University of Padova, 63,Via Trieste, I-35121

Padova, Italy e-mail: giorgiaigroig@alice.it 
although based on different viewpoints, they lead to the same values for the prices in the case when all relevant quantities are fully observable. The second purpose is to extend the two approaches to situations when some of the underlying quantities of the market model are not fully observable and, furthermore, not all observables are also investment instruments and to compare the two approaches also in this latter case.

The first approach is based on work mainly done by E.R.Fernholz and I.Karatzas together with co-workers and of which an overview is given in [2]. We shall refer to it as "Fernholz-Karatzas (FK) approach". The basic idea here is taken from a classical approach in incomplete markets whereby the price of a derivative asset is defined as the smallest superreplicating initial capital. By utilizing the exponential (local) martingale given by the density process $Z(\cdot)$, it is shown that this price can be obtained by utilizing $\frac{Z(\cdot)}{B(\cdot)}$ as "deflator" $(B(t)$ is the money market account/local riskless asset). In this context it is also shown that, even without existence of a martingale measure, the market can be completed and a hedging strategy is explicitly exhibited.

The second approach is based on work done by E.Platen and coworkers and a comprehensive account can be found in the book [5]. Platen calls it the "Benchmark approach" and we shall refer to it under this same name with the acronym (PB). Here the methodology is based on utility indifference pricing thereby showing that the resulting price is independent of the chosen utility function as well as of the initial wealth and it does not require the claim to be replicable. By showing, in this paper, the equivalence of the two approaches in the case of complete observation we also show that, although by (PB) the claim does not have a priori to be replicable, it is so a posteriori and a replicating strategy is given by the one constructed in the (FK) approach.

Since the (FK) approach relies on completing the market, while $(\mathrm{PB})$ is of the utility indifference pricing type that is typically designed for incomplete markets, it appears to be rather intuitive that (FK) has to be restricted to those situations where all observables are also investment instruments. In fact we show that, while in the complete observation case the two approaches are equivalent, there are some differences in the case of incomplete observation if not all observables are also investment instruments.

We consider a classical diffusion type model, although extensions to discontinuous market models may be envisaged. A major role in both approaches is plaid by the growth optimal portfolio (GOP) and we briefly summarize some of its basic features. We also discuss the relationship between the (GOP) strategy and growth rate under complete and incomplete observation. We always assume however that the value of the GOP is observable (possibly via a proxy).

We start in section 2 by describing the model and deriving the GOP. Section 3 is a brief account on how some form of classical arbitrage may arise implying that then an equivalent martingale measure does not exist. It is based on [2], further instances may be found in [5]. In section 4 we summarize the basic features of the two approaches, adding some detail to the proofs of the main results and showing their equivalence under full observation of all relevant quantities. In section 5 
we first describe the incomplete observation model that is based on [7] and where, furthermore, not all observables are also investment instruments and discuss some consequences for the GOP and utility maximization. Then we describe extensions of the two approaches to this model and discuss a possible equivalence.

\section{The Model}

We consider a market $\mathscr{M}$ with a locally riskless asset and a certain number $n$ of risky assets with prices $B(t)$ and $S_{i}(t)$ respectively that, on a given filtered probability space $\left(\Omega, \mathscr{F}, \mathscr{F}_{t}, P\right)$, satisfy the following diffusion-type model

$$
\begin{aligned}
& d B(t)=B(t) r(t) d t, \quad B(0)=1, \\
& d S_{i}(t)=S_{i}(t)\left(b_{i}(t) d t+\sum_{j=1}^{n} \sigma_{i j}(t) d w_{j}(t)\right), \quad S_{i}(0)=s_{i}>0, i=1, \ldots, n .
\end{aligned}
$$

We assume the number of driving Wiener processes $w_{j}(t)$ to be equal to the number of risky assets and that the diffusion matrix $\sigma(t)=\left\{\sigma_{i j}(t)\right\}_{i, j=1, \cdots, n}$ is non singular for each $t \in[0, T]$ with $T<\infty$. Furthermore, the coefficients in (1) are progressively measurable and satisfy

$$
\int_{0}^{T}|r(t)| d t+\sum_{i=1}^{n} \int_{0}^{T}\left(\left|b_{i}(t)\right|+\sum_{j=1}^{n}\left(\sigma_{i j}(t)\right)^{2}\right) d t<\infty \text { a.s. for any } T \in(0, \infty) .
$$

While for most of the results below the filtration $\left\{\mathscr{F}_{t}\right\}$ can be fully general, for some of them we shall, without much loss of generality, assume that $\mathscr{F}_{t}=\mathscr{F}_{t}^{w}$.

From (1) we obtain for the risky assets' log prices

$$
d \log S_{i}(t)=\gamma_{i}(t) d t+\sum_{j=1}^{n} \sigma_{i j}(t) d w_{j}(t), \quad i=1, \ldots, n
$$

with

$$
\gamma_{i}(t):=b_{i}(t)-\frac{1}{2} a_{i i}(t), \quad a(t)=\sigma \sigma^{\prime}(t), i=1, \ldots, n .
$$

Under controlled growth of $a(t)$ as e.g.

$$
\lim _{T \rightarrow \infty}\left(\frac{\log \log T}{T^{2}} \int_{0}^{T} a_{i i}(t) d t\right)=0, \quad \text { a.s. } i=1, \cdots, n
$$

one has

$$
\lim _{T \rightarrow \infty} \frac{1}{T}\left(\log S_{i}(T)-\int_{0}^{T} \gamma_{i}(t) d t\right)=0
$$

which justifies the definition of $\gamma_{i}(t)$ as growth rate of the $i-t h$ asset. 
A portfolio strategy (investment rates) is a progressively measurable process $\pi(t)=\left(\pi_{1}(t), \cdots, \pi_{n}(t)\right)$ with values in

$$
\bigcup_{k \in \mathbb{N}}\left\{\left(\pi_{1}, \cdots, \pi_{n}\right) \in \mathbb{R} \mid \pi_{1}+\cdots+\pi_{n} \leq 1, \pi_{1}^{2}+\cdots+\pi_{n}^{2} \leq k^{2}\right\} .
$$

The ratio $1-\sum_{i=1}^{n} \pi_{i}(t)$ is invested in the risk-free asset.

Let $V^{v, \pi}(t)$ be the value at $t$ of a self financing portfolio with $V^{v, \pi}(0)=v$. From the self financing condition one then has

$$
\begin{aligned}
& \frac{d V^{v, \pi}(t)}{V^{v, \pi}(t)}=\left(1-\sum_{i=1}^{n} \pi_{i}(t)\right) \frac{d B(t)}{B(t)}+\sum_{i=1}^{n} \pi_{i}(t) \frac{d S_{i}(t)}{S_{i}(t)} \\
& =\left(1-\sum_{i=1}^{n} \pi_{i}(t)\right) r(t) d t+\sum_{i=1}^{n} \pi_{i}(t)\left(b_{i}(t) d t+\sum_{j=1}^{n} \sigma_{i j}(t) d w_{j}(t)\right)
\end{aligned}
$$

Putting $\sigma_{\pi j}(t):=\sum_{i=1}^{n} \pi_{i}(t) \sigma_{i j}(t)$, we have, analogously to (3) and (4),

$$
d \log V^{v, \pi}(t)=\gamma_{\pi}(t) d t+\sum_{j=1}^{n} \sigma_{\pi j}(t) d w_{j}(t), \quad i=1, \cdots, n
$$

with the portfolio growth rate

$$
\gamma_{\pi}(t)=r(t)+\sum_{j=1}^{n} \sigma_{\pi j}(t) \theta_{j}(t)-\frac{1}{2}\left(\sum_{j=1}^{n} \sigma_{\pi j}(t)\right)^{2}
$$

where, in vector notation,

$$
\theta(t):=(\sigma(t))^{-1}(b(t)-r(t) \underline{1})
$$

is the "market price of risk". Under a controlled growth of $a(t)$ analogous to (5), also here one has

$$
\lim _{T \rightarrow \infty} \frac{1}{T}\left(\log V^{v, \pi}(T)-\int_{0}^{T} \gamma_{\pi}(t) d t\right)=0
$$

justifying again the name "growth rate" and notice that $V^{v, \pi}=v V^{1, \pi}:=v V^{\pi}$. This latter scaling property of $V$ allows us to derive most of the results for the simpler $V^{\pi}$, they will then hold automatically also for $V^{v, \pi}$ for any $v>0$.

Definition 1 Given an initial wealth $v$, the set of admissible strategies is $\mathscr{H}(v):=$ $\cup_{T>0} \mathscr{H}(v ; T)$ where

$$
\mathscr{H}(v ; T):=\left\{\pi(\cdot) \mid P\left\{V^{v, \pi}(t) \geq 0, \forall 0 \leq t \leq T\right\}=1\right\} .
$$

A major role is plaid by the so-called growth optimal portfolio (GOP) that maximizes the growth rate among the admissible portfolios (it maximizes also the expected log-utility). 
In vector form we have for the growth rate for a generic portfolio $\pi$

$$
\gamma_{\pi}(t)=r(t)+\pi^{\prime}(t) \sigma(t) \theta(t)-\frac{1}{2} \pi^{\prime}(t) \sigma \sigma^{\prime}(t) \pi(t)
$$

which is a concave function of $\pi$ so that, denoting by $\rho(t)$ the GOP strategy that maximizes this growth rate, one has

$$
\rho(t)=\left(\sigma^{\prime}(t)\right)^{-1} \theta(t)
$$

From (8) and (9) we have for any admissible $\pi$

$$
\frac{d V^{\pi}(t)}{V^{\pi}(t)}=r(t) d t+\pi^{\prime}(t) \sigma(t)(\theta(t) d t+d w(t))
$$

which implies for $\pi=\rho$

$$
\frac{d V^{\rho}(t)}{V^{\rho}(t)}=\left(r(t)+\|\theta(t)\|^{2}\right) d t+\theta^{\prime}(t) d w(t)
$$

and so

$$
V^{\rho}(t)=\exp \left[\int_{0}^{t} r(s) d s+\frac{1}{2} \int_{0}^{t}\|\theta(s)\|^{2} d s+\int_{0}^{t} \theta^{\prime}(s) d w(s)\right]
$$

Furthermore, the growth rate of the GOP is

$$
\gamma_{\rho}(t)=r(t)+\frac{1}{2}\|\theta(t)\|^{2}
$$

\section{Relative Arbitrage and Absence of Martingale Measures}

Traditional arbitrage pricing theory is based on equivalent martingale measures. In particular, defining

$$
Z(t):=\exp \left\{-\int_{0}^{t} \theta^{\prime}(s) d w(s)-\frac{1}{2} \int_{0}^{t}\|\theta(s)\|^{2} d s\right\}, 0 \leq t<\infty
$$

with $\theta(t)$ as in (10), the following holds: if $E\{Z(T)\}=1$ for any given $T \in(0, \infty)$, then $Z(t)$ is a mean one martingale and, defining a measure $Q \sim P$ with $\left.\frac{d Q}{d P}\right|_{\mathscr{F}_{T}}=$ $Z(T)$, one has that $\hat{w}(t)=w(t)+\int_{0}^{t} \theta(s) d s$ is a Wiener process on $\left(\Omega, \mathscr{F}, \mathscr{F}_{t}, Q\right)$ and $\bar{S}_{i}(t):=B^{-1}(t) S_{i}(t)$ are $\left(\mathscr{F}_{t}, Q\right)$-martingales.

There are however situations, and below we shall mention some instances, where $E\{Z(T)\}<1$ for some $T \in(0, \infty)$ and then there does not exist an equivalent martingale measure and the traditional pricing approach breaks down. In view of mentioning examples where $E\{Z(T)\}<1$ let us give the following definition (see [2]) 
Definition 2 Given two portfolio strategies $\pi(\cdot)$ and $\rho(\cdot)$, we say that $\pi(\cdot)$ represents an arbitrage opportunity relative to $\rho(\cdot)$ on $[0, T]$ for $T>0$ if, with $V^{\pi}(0)=$ $V^{\rho}(0)$, one has

$$
\mathbb{P}\left(V^{\pi}(T) \geq V^{\rho}(T)\right)=1 \quad \text { and } \quad \mathbb{P}\left(V^{\pi}(T)>V^{\rho}(T)\right)>0 .
$$

One can then show the following proposition (see again [2])

Proposition 1 If model (1) satisfies $\xi^{\prime}\left(\sigma \sigma^{\prime}\right)(t) \xi \leq K\|\xi\|^{2}, \forall \xi \in \mathbb{R}^{n}, \forall t \in[0, \infty)$ with $K>0$ and for a $T>0$ there exists an arbitrage relative to a given portfolio $\rho(\cdot)$, then $Z(t)$ is a strictly local martingale and $E\{Z(T)\}<1$.

As a corollary one can show (see always [2])

Corollary 2 Under the assumptions of Proposition 1 and assuming $\mathscr{F}_{t}=\mathscr{F}_{t}{ }^{w}$, there does not exist an equivalent martingale measure.

In [2] it is shown how one can construct relative arbitrage also on arbitrary time horizons, which leads to one kind of examples where there does not exist an equivalent martingale measure. Other kinds of examples can be found in [5].

Since in the situations corresponding to the above examples the traditional pricing approach based on equivalent martingale measures breaks down, one may wonder what can be done in such situations for what concerns pricing and the rest of the paper is devoted to this issue.

\section{Pricing Under Absence of an Equivalent Martingale Measure (the Case of Complete Observation)}

We summarize here two approaches and show that, although based on different viewpoints, they lead to the same prices.

\subsection{The "Fernholz-Karatzas Approach"}

This subsection in based on [2]. The main idea here is taken from a classical approach in incomplete markets, whereby a reasonable way to assign a price to a derivative asset is to put it equal to the smallest initial amount needed to superreplicate a given claim with a self financing portfolio.

The methodology is based on showing that, even without existence of a martingale measure, the market can be completed and here the proof is constructive by exhibiting a hedging strategy.

One has the following theorem (see [2],[3])

Theorem 3 Consider a given a maturity $T$ and a claim $Y \in \mathscr{F}(T)$ such that $E\left\{Y \frac{Z(T)}{B(T)}\right\} \in(0, \infty)$ with $Z(t)$ as in (18). For $t \in[0, T]$, let 


$$
\mathscr{H}_{t}(v ; T):=\left\{\pi(\cdot) \mid P\left\{V^{v, \pi}(s) \geq 0, \forall t \leq s \leq T\right\}=1 ; V^{v, \pi}(t)=v \in \mathscr{F}(t)\right\}
$$

namely the set of admissible strategies starting from a capital $v$ at time $t$ and put

$$
\mathscr{U}^{Y}(t):=\operatorname{essinf}\left\{v>0 \mid \exists \pi \in \mathscr{H}_{t}(v ; T) \text { s.t. } V^{v, \pi}(T) \geq Y\right\}
$$

which represents the minimal hedging price at time $t$. Let, furthermore,

$$
Y(t):=\frac{B(t)}{Z(t)} E\left\{Y \frac{Z(T)}{B(T)} \mid \mathscr{F}(t)\right\}
$$

with the expectation under the physical measure P. If $\mathscr{F}(t)=\mathscr{F}^{w}(t)$, then

$$
Y(t)=\mathscr{U}^{Y}(t)
$$

Remark 4 On the basis of this theorem we shall now take $Y(t)$ as price of the claim at time $t$. Notice that in formula (20) the process $\frac{Z(t)}{B(t)}$ acts as a "deflator". Notice furthermore that, if $Z(t)$ is a true martingale and $\mathscr{S}$ is the set of martingale measures then, since $\mathscr{U}^{Y}(t)$ is the minimal superhedging price,

$$
Y(t)=\mathscr{U}^{Y}(t)=\sup _{Q \in \mathscr{S}} E^{Q}\left\{\frac{B(t)}{B(T)} Y \mid \mathscr{F}_{t}\right\}
$$

namely the here defined price coincides with the standard superhedging price.

Proof. (of Thm. 3) We proceed along several steps, whereby we shall also need the fact that, as will be shown in Section 4.3 below, $V^{\rho}(t)=\frac{B(t)}{Z(t)}$.

i) Notice first that from (14) and (15) we have for $\hat{V}^{v, \pi}(s):=\frac{V^{v, \pi}(s)}{V^{\rho}(s)}=\frac{Z(s)}{B(s)} V^{v, \pi}(s)$, with $s \in[t, T]$ and any $v>0$, that (in vector form)

$$
d \hat{V}^{v, \pi}(s)=\hat{V}^{v, \pi}(s)\left(\pi^{\prime}(s) \sigma(s)-\theta^{\prime}(s)\right) d w(s)
$$

so that $\hat{V}^{v, \pi}$ is a non-negative local martingale and therefore a supermartingale with $\hat{V}^{v, \pi}(t)=v$ (recall that $V^{\rho}(s)$ is the GOP with $\left.V^{\rho}(t)=1\right)$. Since, as will be shown in iii) below, the set in the definition of $\mathscr{U}^{Y}(t)$ is not empty, then there exists $\pi \in \mathscr{H}_{t}(v ; T)$ such that $V^{v, \pi}(T) \geq Y$ and so

$$
\begin{aligned}
Y(t) & =\frac{B(t)}{Z(t)} E\left\{Y \frac{Z(T)}{B(T)} \mid \mathscr{F}(t)\right\} \leq \frac{B(t)}{Z(t)} E\left\{V^{v, \pi}(T) \frac{Z(T)}{B(T)} \mid \mathscr{F}(t)\right\} \\
& \leq \frac{B(t)}{Z(t)} V^{v, \pi}(t) \frac{Z(t)}{B(t)}=v .
\end{aligned}
$$

which implies $Y(t) \leq \mathscr{U}^{Y}(t)$.

To complete the proof, in the next steps we show that $Y(t) \geq \mathscr{U}^{Y}(t)$ and we do it by explicitly constructing a hedging strategy.

ii) Under the assumptions of the theorem we can define the $(P, \mathscr{F}(t))$-martingale 


$$
M(s):=E\left\{Y \frac{Z(T)}{B(T)} \mid \mathscr{F}(s)\right\}
$$

which, given that $\mathscr{F}(t)=\mathscr{F}^{w}(t)$, admits the following "martingale representation" for $s \in[t, T]$,

$$
M(s)=M(t)+\int_{t}^{s} \psi^{\prime}(u) d w(u)=Y(t) \frac{Z(t)}{B(t)}+\int_{t}^{s} \psi^{\prime}(u) d w(u)
$$

for a progressively measurable, a.s. square integrable process $\psi(t)$ and where the last equality follows from the definition of $Y(t)$ in (20)

iii) If we choose $v$ such that

$$
v=\hat{V}^{v, \pi}(t)=M(t)=E\left\{Y \frac{Z(T)}{B(T)} \mid \mathscr{F}(t)\right\}
$$

and $\pi$ such that

$$
d \hat{V}^{v, \pi}(s)=d M(s) \text { for } s \in[t, T]
$$

then $\hat{V}^{v, \pi}(s)=M(s)$ for all $s \in[t, T]$ and we have

$$
V^{v, \pi}(s)=V^{\rho}(s) M(s)=\frac{B(s)}{Z(s)} E\left\{Y \frac{Z(T)}{B(T)} \mid \mathscr{F}(s)\right\}
$$

This implies that

$$
\left\{\begin{array}{l}
v=V^{v, \pi}(t)=Y(t) \\
V^{v, \pi}(T)=Y
\end{array}\right.
$$

i.e. the strategy $\pi$ satisfying (25) is an admissible hedging strategy and this also implies that the set in the definition of $\mathscr{U}^{Y}(t)$ is not empty and so $Y(t) \geq \mathscr{U}^{Y}(t)$.

iv) It remains to explicitly exhibit an admissible strategy $\pi$ satisfying (25). It suffices to equalize the coefficients of $d w(t)$ in (22) and (24) namely, recalling that $\hat{V}^{v, \pi}(s):=\frac{V^{v, \pi}(s)}{V^{\rho}(s)}=\frac{Z(s)}{B(s)} V^{v, \pi}(s)=M(s)$, to impose the following

$$
\frac{Z(s)}{B(s)} V^{v, \pi}(s)\left(\pi^{\prime}(s) \sigma(s)-\theta^{\prime}(s)\right)=\psi^{\prime}(s)
$$

which leads to

$$
\pi(s)=\sigma^{-1}(s)\left(\frac{\psi(s)}{M(s)}+\theta(s)\right)
$$

for $s \in[t, T]$ with $t \in[0, T]$. 


\subsection{The “Benchmark Approach" of Platen}

This subsection is based on [5]. Here the methodology builds on utility indifference pricing thereby showing that the resulting price is independent of the chosen utility function and of the initial wealth and it does not require the claim to be replicable.

To simplify the presentation, in what follows we shall denote with a "bar" the values expressed in units of the locally riskless asset, for example

$$
\bar{V}^{v, \pi}(t)=B^{-1}(t) V^{v, \pi}(t)
$$

We start with the following

Definition 3 An asset or portfolio value expressed in units of the GOP is called benchmarked. A price or portfolio process is fair if its benchmarked value is a $\left(\mathscr{F}_{t}, P\right)$-martingale.

This definition corresponds to the property of a discounted self financing portfolio value process to be a martingale under any martingale measure, provided there exists one. Notice also that it is reasonable to restrict oneself to fair portfolios because they are martingales and a martingale is the smallest among the supermartingales that reach a same value at a future (stopping) time.

Utility indifference pricing is based on expected utility maximization, which we recall next. Given a utility function $U(\cdot)$ and an initial capital $v>0$, the utility maximization problem consists in determining an admissible strategy $\tilde{\pi}$ such that

$$
E\left\{U\left(\bar{V}^{v, \tilde{\pi}}(T)\right)\right\} \geq E\left\{U\left(\bar{V}^{v, \pi}(T)\right)\right\} \quad \forall \pi \in \mathscr{H}(v ; T)
$$

Here we want the strategies also to generate a fair portfolio process.

A rather straightforward extension of the so-called "martingale approach" to utility maximization (see e.g. [8]), coupled with fairness of the corresponding portfolio process, leads to the following

Lemma 5 Given a utility function $U(\cdot)$ and an initial wealth $v>0$, the fair discounted terminal portfolio value solving (28) is given by

$$
\bar{V}^{v, \tilde{\pi}}(T)=\dot{U}^{-1}\left(\frac{\lambda}{\bar{V}^{v, \rho}(T)}\right)
$$

where $\dot{U}$ denotes the derivative of $U, \bar{V}^{v, \rho}(T)$ is the discounted terminal value of the GOP and the (Lagrange) parameter $\lambda$ is determined from the "budget equation"

$$
E\left\{\frac{V^{v, \tilde{\pi}}(T)}{V^{v, \rho}(T)}\right\}=\frac{V^{v, \tilde{\pi}}(0)}{V^{v, \rho}(0)}=\frac{v}{v}=1
$$

The strategy $\tilde{\pi}$ is any admissible strategy leading to the terminal portfolio value in (29). 
Remark 6 In the standard martingale approach, where the existence of martingale measure is given, the budget equation results from the martingality of the discounted value process of a self financing portfolio. Not assuming here the existence of a martingale measure, we impose the condition of fairness instead.

Proof. (Sketch of the proof).

The budget equation (30) is a consequence of the requirement of fairness. Given the budget constraint, by the Lagrange multiplier method the problem becomes that of maximizing

$$
E\left\{U\left(\bar{V}^{v, \pi}(T)\right)-\lambda \frac{V^{v, \pi}(T)}{V^{v, \rho}(T)}\right\}
$$

The "martingale approach" now consists in maximizing

$$
U(\bar{V})-\lambda \frac{V}{V^{v, \rho}(T)}=U(\bar{V})-\lambda \frac{\bar{V}}{\bar{V}^{v, \rho}(T)}
$$

with respect to $\bar{V}$ that leads, for each "scenario" $\omega \in \Omega$, to the optimal terminal value $\bar{V}^{v, \tilde{\pi}}(T)$ given by (29). The strategy $\tilde{\pi}$ is then determined as the hedging strategy for the "claim" $\bar{V}^{v, \tilde{\pi}}(T)$.

We come next to the actual utility indifference pricing. Given $U(\cdot)$ and the corresponding $\tilde{\pi}$, it is well known that the utility indifference price $P(t)$ at time $t$ of a given claim $Y$ is such that, defining

$$
p_{\varepsilon, P(t)}^{v, \tilde{\pi}}:=E\left\{U\left[(V(t)-\varepsilon P(t)) \frac{\bar{V}^{v, \tilde{\pi}}(T)}{V(t)}+\varepsilon \bar{Y}\right] \mid \mathscr{F}(t)\right\}
$$

where $V(t)$ is the investor's wealth at time $t$ starting from $v$ and following the strategy $\tilde{\pi}$, one has

$$
\lim _{\varepsilon \rightarrow 0} \frac{p_{\varepsilon, P(t)}^{v, \tilde{\pi}}-p_{0, P(t)}^{v, \tilde{\pi}}}{\varepsilon}=0 \quad \text { a.s }
$$

Using a Taylor expansion of $p_{\varepsilon, P(t)}^{v, \tilde{\pi}}$ around $p_{0, P(t)}^{v, \tilde{\pi}}$, it is easily seen that this leads to the following (see [5])

Proposition 7 Given $U(\cdot)$ and the corresponding $\tilde{\pi}$, the utility indifference price is

$$
P(t)=\frac{E\left\{\dot{U}\left(\bar{V}^{v, \tilde{\pi}}(T)\right) \bar{Y} \mid \mathscr{F}(t)\right\}}{E\left\{\dot{U}\left(\bar{V}^{v, \tilde{\pi}}(T)\right) \frac{\bar{V}^{v, \tilde{\pi}}(T)}{V(t)} \mid \mathscr{F}(t)\right\}} .
$$

where, again, $\dot{U}$ denotes the derivative of $U$.

We can now show the following (see [5], [3])

Theorem 8 The utility indifference price is given by

$$
P(t)=V^{\rho}(t) E\left\{\frac{Y}{V^{\rho}(T)} \mid \mathscr{F}(t)\right\}
$$


where $V^{\rho}(t)=V^{1, \rho}(t)$ is the portfolio value corresponding to the GOP strategy $\rho$.

Proof. Substituting (29) into (33) results in the following chain of equalities, where we use the fairness property of the benchmarked portfolio values

$$
\begin{aligned}
P(t) & =\frac{E\left\{\frac{Y}{V^{v, \rho}(T)} \mid \mathscr{F}(t)\right\}}{E\left\{\frac{V^{\prime, \tilde{n}}(T)}{V^{v, \rho}(T)} \frac{1}{V(t)} \mid \mathscr{F}(t)\right\}}=\frac{V^{v, \tilde{\pi}}(t) E\left\{\frac{Y}{V^{v, \rho}(T)} \mid \mathscr{F}(t)\right\}}{\frac{V^{v, \tilde{\pi}}(t)}{V^{v, \rho}(t)}} \\
& =V^{v, \rho}(t) E\left\{\frac{Y}{V^{v, \rho}(T)} \mid \mathscr{F}(t)\right\}=v V^{\rho}(t) E\left\{\frac{Y}{V^{\rho}(T)} \mid \mathscr{F}(t)\right\} \\
& =V^{\rho}(t) E\left\{\frac{Y}{V^{\rho}(T)} \mid \mathscr{F}(t)\right\}
\end{aligned}
$$

whereby the Lagrange parameter as well as the initial wealth $v$ have canceled out.

Remark 9 Formula (34) corresponds to a standard arbitrage pricing formula, whereby one takes as numeraire the GOP and the resulting martingale measure is the physical measure P. It is thus an instance of the so-called "real world pricing". In line with Definition 3 the price $P(t)$ is a fair price. We stress once again that the pricing formula (34) is independent of the utility function $U(\cdot)$ and of the initial wealth $v>0$ and does not require the existence of a martingale measure nor the claim to be a priori replicable. The claim will though turn out to be replicable a posteriori via the equivalence of the pricing approach of this subsection 4.2 with that of the previous subsection 4.1 that will be discussed next.

\subsection{Equivalence of the Two Pricing Approaches}

Recall that, according to the pricing approach in subsection 4.1, the (superhedging) price $Y(t)$ at time $t \in[0, T]$ of a given claim $Y$ with maturity $T$ is given by (see (20))

$$
Y(t):=\frac{B(t)}{Z(t)} E\left\{Y \frac{Z(T)}{B(T)} \mid \mathscr{F}(t)\right\}
$$

and it corresponds to that of the standard martingale approach if $Z(t)$ is a true martingale. On the other hand, the (utility indifference) price $P(t)$ according to the approach in subsection 4.2 is given by (see (34))

$$
P(t)=V^{\rho}(t) E\left\{\frac{Y}{V^{\rho}(T)} \mid \mathscr{F}(t)\right\}
$$

We now show that the two pricing approaches lead to the same result by proving

Proposition 10 One has

$$
Y(t)=P(t)
$$


Proof. It suffices to show that

$$
V^{\rho}(t)=\frac{B(t)}{Z(t)}
$$

which is equivalent to showing that the discounted GOP value is the inverse of the "density process" $Z(t)$ a fact that is generally known under standard assumptions. We show it here formally within our context. Recalling (16), namely

$$
V^{\rho}(t)=\exp \left[\int_{0}^{t} r(s) d s+\frac{1}{2} \int_{0}^{t}\|\theta(s)\|^{2} d s+\int_{0}^{t} \theta^{\prime}(s) d w(s)\right]
$$

and noticing that from (18) one has

$$
\begin{aligned}
\frac{Z(t)}{B(t)} & =\frac{\exp \left[-\int_{0}^{t} \theta^{\prime}(s) d w(s)-\frac{1}{2} \int_{0}^{t}\|\theta(s)\|^{2} d s\right]}{\exp \left[\int_{0}^{t} r(s) d s\right]} \\
& =\exp \left[-\int_{0}^{t} \theta^{\prime}(s) d w(s)-\frac{1}{2} \int_{0}^{t}\|\theta(s)\|^{2} d s-\int_{0}^{t} r(s) d s\right\}
\end{aligned}
$$

the result follows immediately.

Remark 11 Notice that none of the two approaches, that in subsection 4.1 and that in 4.2, requires the existence of an equivalent martingale measure. From the first approach it is easily seen that, if $Z(t)$ is a martingale and therefore there exists an equivalent martingale measure, then the usual arbitrage (martingale-based) price coincides with that in subsection 4.1 and, by the equivalence just shown, also with that in subsection 4.2. The pricing formula in subsection 4.2 and, by the equivalence, also that in subsection 4.1, corresponds to that of a standard pricing approach by taking as numeraire the GOP, for which the physical measure is a martingale measure (this also justifies the fact that none of the two approaches required the existence of a martingale measure different from the physical measure P). The approach in subsection 4.2 does not require the claim to be a priori replicable. The constructive approach of subsection 4.1, which is based on a replication argument and exhibits explicitly a replication strategy, implies, via the equivalence, that also in the approach of subsection 4.2 the claim is in fact replicable. We finally want to point out that the equivalence of the two approaches is implicit in some parts of [5], for example in section 11.5 where it is emphasized that "for a nonreplicable payoff the real world pricing formula provides the minimal nonnegative price process" or also in later sections like 12.2 where Fig. 12.2.4 is intended to "visualize the fact that fair prices are the minimal prices that replicate a contingent claim" and for this the authors refer to Corollary 10.4.2 (always in [5]). 


\section{Pricing Under Absence of an Equivalent Martingale Measure (the Case of Incomplete Observation)}

We now extend the results, summarized in the previous section 4 for the case of complete observation on the market model, to a market model with incomplete observation. We first describe the model that we are considering.

\subsection{The Model}

The model is taken from [7]. Consider the model (1) with one riskless asset and $n$ risky assets and assume that the observable quantities are the $n+1$ prices of the primary assets, the empirical covariances of the risky assets' log-prices with that of the GOP (we assume that the values of the GOP or of a proxy thereof are observable) in addition to the short rate of interest $r(t)$. To simplify the notation, in what follows we shall consider all the prices expressed in units of the riskless asset (discounted values) and, whenever ambiguity may arise, denote their values with a bar. Let

$$
C(t)=\left(C_{1}(t), \cdots, C_{2 n}(t)\right)
$$

be the vector of observable (discounted) quantities (excluding the short rate). It is natural to assume that the underlying filtration $\mathscr{F}(t)$ measures $C(t)$. Assume then that there are also a certain number of unobservable quantities, denote them by

$$
A(t)=\left(A_{1}(t), \cdots, A_{m}(t)\right)
$$

that we may think of as representing latent factors that affect the primary assets' price dynamics. We restrict the volatility matrix $\sigma(t)$ to depend only on the observable quantities and to stress this fact we shall write $\sigma(t)=\sigma(t, C(t))$ assuming furthermore that it is invertible for all $t \leq T<\infty$. Recalling the market price of risk in (10), here we then write it as

$$
\theta(t, A(t))=(\sigma(t, C(t)))^{-1}(b(t, A(t))-r(t) \underline{1})
$$

to stress the dependence also on $A(t)$ (it depends also on $C(t)$ not only through $\sigma(t, C(t))$ but in general also through $b(\cdot)$ that is $\mathscr{F}(t)-$ measurable).

In line with the above definitions we rewrite the dynamics of the discounted values of the risky assets as (see (1))

$$
d \bar{S}_{i}(t)=\bar{S}_{i}(t)\left[\sum_{j=1}^{n} \sigma_{i j}(t, C(t))\left(\theta_{j}(t, A(t)) d t+d w_{j}(t)\right)\right], \quad \bar{S}_{i}(0)=s_{i}>0
$$

and the discounted value process $\bar{V}^{v, \pi}(t)=v \bar{V}^{1, \pi}(t)=v \bar{V}^{\pi}(t)$ of a self financing portfolio (that invests only in the risky assets) becomes (see (14)) 


$$
\frac{d \bar{V}^{\pi}(t)}{\bar{V}^{\pi}(t)}=\pi^{\prime}(t) \sigma(t, C(t))[\theta(t, A(t)) d t+d w(t)]
$$

Finally, corresponding to (15)

$$
\frac{d \bar{V}^{\rho}(t)}{\bar{V}^{\rho}(t)}=\|\theta(t, A(t))\|^{2}+\theta^{\prime}(t, A(t)) d w(t)
$$

Having assumed that also the empirical log-covariances are observable, we need a model for these quantities as well, and we shall represent them as noisy perturbations of the corresponding unobservable theoretical covariances.

Letting $\Delta$ denote the discrete time observation step and $t_{\ell}:=\ell \Delta$ the grid points of the observations, for the $i-$ th log-covariance we have

$$
\begin{aligned}
z_{i}(t) & =\left[\ln \left(\bar{S}_{i}\right), \ln \left(\bar{V}^{\rho}\right)\right]_{\Delta, t} \\
& =\sum_{l=1}^{\left[\frac{t}{\Delta}\right]}\left(\ln \left(\bar{S}_{i}\left(t_{l}\right)\right)-\ln \left(\bar{S}_{i}\left(t_{l-1}\right)\right)\right)\left(\ln \left(\bar{V}^{\rho}\left(t_{l}\right)\right)-\ln \left(\bar{V}^{\rho}\left(t_{l-1}\right)\right)\right)
\end{aligned}
$$

where $[a]$ denotes the largest integer less than or equal to $a$. Putting

$$
D(t)=\left(D_{1}(t), \cdots, D_{2 n+m}(t)\right)^{\prime}=\left(C_{1}(t), \cdots, C_{2 n}(t), A_{1}(t), \cdots, A_{m}(t)\right)^{\prime}
$$

and

$$
B_{i}(t, D(t)):=\sum_{j=1}^{n} \sigma_{i j}(t, C(t)) \theta_{j}(t, A(t))
$$

the $i-$ th theoretical covariance is then

$$
\left[\ln \left(\bar{S}_{i}\right), \ln \left(\bar{V}^{\rho}\right)\right]_{t}=\left\langle\ln \left(\bar{S}_{i}\right), \ln \left(\bar{V}^{\rho}\right)\right\rangle_{t}=\int_{0}^{t} B_{i}(s, D(s)) d s
$$

Since the theoretical covariances are the limit in probability of the empirical covariances, we may postulate for the latter the following model

$$
d z_{i}(t)=B_{i}(t, D(t)) d t+d \eta_{i}(t)
$$

where $\eta_{i}(t)$ are $n$ independent Wiener processes, independent of the $w_{j}(t)$, and they represent the discrepancy between theoretical and empirical covariances.

\subsection{Corresponding Complete Observation Model}

Since the unobservable factors are inputs to the model, also solutions of problems for the model will depend on them. On the other hand prices and strategies should only depend on the available information that results from the observations. In the following two subsections we shall show that, for our pricing problem, we can 
equivalently consider a complete observation problem corresponding to the original one under incomplete observation and in this equivalent problem all inputs are given by quantities that are all observable either directly or indirectly. In this subsection we now derive this equivalent complete observation problem, sometimes also referred to as "separated problem".

Put, with some abuse of notation

$$
C(t)=\left(\bar{S}_{1}(t), \cdots, \bar{S}_{n}(t), z_{1}(t), \cdots, z_{n}(t)\right)^{\prime}
$$

that now represents the totality of the observable quantities and consider the $2 n$-dimensional Wiener process

$$
W(t)=\left(w_{1}(t), \cdots, w_{n}(t), \eta_{1}(t), \cdots, \eta_{n}(t)\right)^{\prime}
$$

Recalling (40) and (41) and putting

$$
B(t, D(t))=\left(B_{1}(t, D(t)), \cdots, B_{n}(t, D(t))\right)^{\prime}
$$

we obtain the following dynamics for the global $2 n$-dimensional vector $C(t)$

$$
\begin{aligned}
d C(t) & =\left(\begin{array}{l}
\operatorname{diag}(\bar{S}(t)) B(t, D(t)) \\
B(t, D(t))
\end{array}\right) d t+\left(\begin{array}{ll}
\operatorname{diag}(\bar{S}(t)) \sigma(t, C(t)) & 0 \\
0 & \mathbf{I}
\end{array}\right) d W(t) \\
& :=F(t, D(t)) d t+G(t, C(t)) d W(t)
\end{aligned}
$$

thereby implicitly defining the (vector) drift $F(t, D)$ and (matrix) volatility $G(t, C)$.

The information filtration is the one generated by the $k:=2 n$ components of $C(t)$ and to stress the "dimension" $k$ of the observations we write

$$
\mathscr{F}^{k}(t):=\sigma\{C(s), s \leq t\}
$$

As it is typically done in partial observation problems, we consider now instead of the unobservable quantities $A(t)$ their conditional distribution (the so-called filter distribution)

$$
p\left(A(t) \mid \mathscr{F}^{k}(t)\right)=p(A(t) \mid \sigma\{C(s), s \leq t\})
$$

We make the following

Assumption 12 The filter for $A(t)$ given $\mathscr{F}^{k}(t)$ is finite-dimensional, namely the filter distribution $p\left(A(t) \mid \mathscr{F}^{k}(t)\right)$ is parametrized by a finite number of parameters

$$
\xi(t)=\left(\xi_{1}(t), \cdots, \xi_{q}(t)\right)^{\prime}
$$

More precisely, $p\left(A(t) \mid \mathscr{F}^{k}(t)\right)=p\left(A(t) ; \xi_{1}(t), \cdots, \xi_{q}(t)\right)$

Remark 13 Well known finite dimensional filters are the Kalman-Bucy filter and the Wonham filter for the case when the unobserved quantities form a finite-state Markov chain. More generally, the Extended Kalman filter is a finite-dimensional 
filter that is applicable, modulo an approximation, to a variety of situations. In all the above cases the filter parameters form a Markov process driven by the innovations (for the definition of "innovations" see Remark 15 below). This, together with (46) motivates and also justifies the following additional assumption.

Assumption 14 By analogy to (40) put

$$
\tilde{D}(t)=\left(C_{1}(t), \cdots, C_{2 n}(t), \xi_{1}(t), \cdots, \xi_{q}(t)\right)^{\prime}
$$

that represents the extended $(2 n+q)$-dimensional vector of "observables". We assume that the parameters $\xi(t)$ of the finite-dimensional filter satisfy

$$
d \xi(t)=H(t, \tilde{D}(t)) d t+K(t, \tilde{D}(t)) d C(t)
$$

We now apply Theorem 7.12 in [4] (see also Proposition 2.3 in [6]) according to which the $k=2 n$-dimensional process $C(t)$ satisfies, instead of (46) where the factor $D(t)$ in the drift is not fully observable, the following dynamics

$$
d C(t)=\tilde{F}(t, \tilde{D}(t)) d t+G(t, C(t)) d \tilde{W}(t)
$$

with

$$
\begin{aligned}
\tilde{F}(t, \tilde{D}(t)) & =E\left\{F(t, D(t)) \mid \mathscr{F}^{k}(t)\right\} \\
& =\int F(t, C(t), A) d p\left(A \mid \mathscr{F}^{k}(t)\right)=\int F(t, C(t), A) d p\left(A ; \xi_{1}, \cdots, \xi_{q}\right)
\end{aligned}
$$

and where $\tilde{W}(t)$ is a $k$-dimensional $\mathscr{F}^{k}-$ Wiener process (with independent components) satisfying

$$
d \tilde{W}(t)=\left(G(t, C(t))^{-1}[d C(t)-\tilde{F}(t, \tilde{D}(t)) d t]\right.
$$

Remark 15 Due to the representation in (53), the process $\tilde{W}(t)$ is also called "innovations process" and notice that by this same (53), combined with (50), the dynamics of the finite-dimensional filter parameters $\xi(t)$ can be expressed in terms of these innovations (see also the last part of Remark 13). Furthermore, from (51) and (53) and recalling the definition of $\mathscr{F}^{k}(t)$ in (47), one has that $\mathscr{F}^{k}(t)=\mathscr{F}^{\tilde{W}}(t)$, namely the observation filtration is equivalently generated by the innovations $\tilde{W}(t)$.

Remark 16 In what follows we shall use the symbol $\tilde{w}(t)$ to denote the $n$-dimensional subvector of the $k=2 n$ dimensional vector $\tilde{W}(t)$, which is formed by the first $n$ components and which (see (51) and the definition of $G(t, C)$ in (46)) satisfies

$$
d \tilde{w}(t)=\left(\sigma(t, C(t))^{-1}\left[(\operatorname{diag}(\bar{S}(t)))^{-1} d \bar{S}(t)-\tilde{B}(t, \tilde{D}(t)) d t\right]\right.
$$

where, by analogy to $\tilde{F}(t, \tilde{D}(t))$ in (52), 


$$
\begin{aligned}
\tilde{B}(t, \tilde{D}(t)) & =E\left\{B(t, D(t)) \mid \mathscr{F}^{k}(t)\right\} \\
& =\int B(t, C(t), A) d p\left(A \mid \mathscr{F}^{k}(t)\right)=\int B(t, C(t), A) d p\left(A ; \xi_{1}, \cdots, \xi_{q}\right)
\end{aligned}
$$

Combining (51) with (50), it is easily seen that, for appropriate coefficients $\alpha(\cdot)$ and $\beta(\cdot)$ one obtains for the extended $(2 n+q)$-dimensional observation vector dynamics of the form

$$
d \tilde{D}_{i}(t)=\alpha_{i}(t, \tilde{D}(t)) d t+\sum_{j=1}^{2 n} \beta_{i j}(t, \tilde{D}(t)) d \tilde{W}_{j}(t)
$$

We shall next derive particularized dynamics, in terms of the innovations, for the (discounted) prices and the GOP.

\subsubsection{Price Dynamics}

The (discounted) prices of the risky assets form (see (49) and (44)) the first $n$ components of $\tilde{D}(t)$ and their dynamics are affected only by the first $n$ components of $\tilde{W}(t)$, the vector of which we had denoted by $\tilde{w}(t)$. Recalling then (41) and (46) as well as (51), one obtains for the discounted asset prices

$$
d \bar{S}_{i}(t)=\bar{S}_{i}(t)\left[\sum_{j=1}^{n} \sigma_{i j}(t, C(t))\left(\tilde{\theta}_{j}(t, \tilde{D}(t)) d t+d \tilde{w}_{j}(t)\right)\right], \bar{S}_{i}(0)=s_{i}>0
$$

where

$$
\tilde{\theta}_{j}(t, \tilde{D}(t))=E\left\{\theta_{j}(t, A(t)) \mid \mathscr{F}^{k}(t)\right\}=\int \theta_{j}(t, A) d p\left(A ; \xi_{1}, \cdots, \xi_{q}\right)
$$

(Recall from the comment after (35) that $\theta(t, A(t))$ hides a possible dependence also on $C(t)$ ). Notice that joining (56) to (57), one obtains a Markovian system and in (57) all the coefficients depend on observable quantities.

\subsubsection{GOP Dynamics}

Given its importance in our analysis, we now want to obtain also for the GOP dynamics analogous to (57). For this purpose notice first that, with price dynamics (57) instead of (36), the dynamics (37) for the discounted portfolio values become (we use $\bar{V}^{\pi, k}$ to stress the information level/observation dimension $k$ )

$$
\frac{d \bar{V}^{\pi, k}(t)}{\bar{V}^{\pi, k}(t)}=\pi^{\prime}(t) \sigma(t, C(t))[\tilde{\theta}(t, \tilde{D}(t)) d t+d \tilde{w}(t)]
$$


where $\pi(t)$ has now to be adapted to $\mathscr{F}^{k}(t)$. This leads to the following adaptation of the definition of the set of admissible strategies, namely (see Definition 1)

Definition 4 Given an initial wealth $v$, the set of admissible strategies under partial observation of level $k$ is $\mathscr{H}^{k}(v):=\bigcup_{T>0} \mathscr{H}^{k}(v ; T)$ where

$$
\mathscr{H}^{k}(v ; T):=\left\{\pi(\cdot) \in \mathscr{F}^{k}(\cdot) \mid P\left\{V^{v, \pi, k}(t) \geq 0, \forall 0 \leq t \leq T\right\}=1\right\} .
$$

By analogy with the case of complete observations (see (9) and notice that now we use discounted values), the growth rate of a portfolio $\pi$ is here

$$
\gamma_{\pi}^{k}(t)=\pi^{\prime}(t) \sigma(t, C(t)) \tilde{\theta}(t, \tilde{D}(t))-\frac{1}{2} \pi^{\prime}(t) \sigma(t, C(t)) \sigma^{\prime}(t, C(t)) \pi(t)
$$

and it leads to the following GOP strategy

$$
\rho^{k}(t)=\left(\sigma^{\prime}(t, C(t))\right)^{-1} \tilde{\theta}(t, \tilde{D}(t))
$$

Substituting the expression for $\rho^{k}(t)$ into (59) we immediately obtain the

Proposition 17 The discounted value of the GOP (with unit initial wealth) satisfies

$$
\frac{d \bar{V}^{\rho, k}(t)}{\bar{V}^{\rho, k}(t)}=\|\tilde{\boldsymbol{\theta}}(t, \tilde{D}(t))\|^{2} d t+\tilde{\boldsymbol{\theta}}^{\prime}(t, \tilde{D}(t)) d \tilde{w}(t)
$$

and the growth rate of the GOP is given by

$$
\gamma_{\rho}^{k}(t)=\frac{1}{2}\|\tilde{\theta}(t, \tilde{D}(t))\|^{2}
$$

\subsubsection{GOP Strategy and Growth Rate Under Complete and Incomplete Observation}

Comparing the expressions for the GOP strategy and growth rate under complete observations (see (13) and (17)) with those under incomplete observations in (62) and (64) (notice that the growth rate under incomplete observation was derived for discounted values so that in (64) the additive term $r(t) \in \mathscr{F}^{k}(t)$ is missing) we obtain

Corollary 18 We have

$$
\begin{aligned}
& \text { i) } \quad \rho^{k}(t)=E\left\{\rho(t) \mid \mathscr{F}^{k}(t)\right\} \\
& \text { ii) } \quad \gamma_{\rho}^{k}(t) \leq E\left\{\gamma_{\rho}(t) \mid \mathscr{F}^{k}(t)\right\}
\end{aligned}
$$

Proof. i) is immediate considering the definition of $\tilde{\theta}(t, \tilde{D})$ in (58), while for ii) we use Jensen's inequality and the fact that, under full observation, we have simply written $\theta_{i}(t)$ for what under incomplete observations is $\theta(t, A(t))$. We thus obtain 
(for the definition of $\tilde{\theta}_{i}(t, \tilde{D}(t))$ see (58))

$$
\begin{aligned}
& E\left\{\gamma_{\rho}^{k}(t)\right\}=\frac{1}{2} \sum_{i=1}^{n} E\left\{\left(\tilde{\theta}_{i}(t, \tilde{D}(t))\right)^{2}\right\}=\frac{1}{2} \sum_{i=1}^{n} E\left\{\left(E\left\{\theta_{i}(t, A(t)) \mid \mathscr{F}^{k}(t)\right\}\right)^{2}\right\} \\
& \leq \frac{1}{2} \sum_{i=1}^{n} E\left\{E\left\{\theta_{i}^{2}(t, A(t)) \mid \mathscr{F}^{k}(t)\right\}\right\}=E\left\{E\left\{\frac{1}{2} \sum_{i=1}^{n} \theta_{i}^{2}(t, A(t)) \mid \mathscr{F}^{k}(t)\right\}\right\} \\
& =E\left\{E\left\{\gamma_{\rho}(t) \mid \mathscr{F}^{k}(t)\right\}\right\}=E\left\{\gamma_{\rho}(t)\right\}
\end{aligned}
$$

\subsubsection{Expected Utility Maximization Under Complete and Incomplete Observation}

Here we furthermore point out that, whenever one has to solve a problem for a partial observation model where the strategies have to be adapted to the filtration representing the available information, then the original problem can equivalently be solved by solving it for the corresponding complete observation model. As an example, which will be relevant for Platen's benchmark approach, we show that expected utility for the original incomplete observation problem is equivalent to the same problem for the corresponding complete observation model.

The original problem can, for an arbitrary initial capital $v>0$, be formulated as (see Definition 4 for the class of admissible strategies and (37) for the portfolio dynamics)

$$
\left\{\begin{array}{l}
\max _{\pi \in \mathscr{H}^{k}(v ; T)} E\left\{U\left(\bar{V}^{v, \pi}(T)\right)\right\} \\
\frac{d \overline{\bar{V}}^{v}, \pi(t)}{\bar{V}^{v, \pi(t)}}=\pi^{\prime}(t) \sigma(t, C(t))[\theta(t, A(t)) d t+d w(t)]
\end{array}\right.
$$

For the corresponding complete observation model the problem becomes (see (59) for the portfolio dynamics)

$$
\left\{\begin{array}{l}
\max _{\pi \in \mathscr{H}^{k}(v ; T)} E\left\{U\left(\bar{V}^{v, \pi, k}(T)\right)\right\} \\
\frac{d \bar{\nu}^{v, \pi, k}(t)}{\bar{V}^{v, \pi, k}(t)}=\pi^{\prime}(t) \sigma(t, C(t))[\tilde{\theta}(t, \tilde{D}(t)) d t+d \tilde{w}(t)]
\end{array}\right.
$$

We show the following

\section{Lemma 19}

$$
\max _{\pi \in \mathscr{H}^{k}(v ; T)} E\left\{U\left(\bar{V}^{v, \pi}(T)\right)\right\}=\max _{\pi \in \mathscr{H}^{k}(v ; T)} E\left\{U\left(\bar{V}^{v, \pi, k}(T)\right)\right\}
$$

Proof. From (53) and (46) we obtain

$$
d \tilde{W}(t)=\left(G(t, C(t))^{-1}[F(t, D(t))-\tilde{F}(t, \tilde{D}(t))] d t+d W(t)\right.
$$


Since $V^{v, \pi}(t)$ and $\bar{V}^{v, \pi, k}(t)$ are driven by $w(t)$ and $\tilde{w}(t)$ respectively, recalling the definitions of $F(\cdot), G(\cdot)$ and $\tilde{F}(\cdot)$ in (46) and (52) as well as the fact that the first $n$ components of $C(t)$ are (see (44)) the discounted prices $\bar{S}_{i}(t)$, we have

$$
\begin{aligned}
d \tilde{w}(t) & =(\sigma(t, C(t)))^{-1}(\operatorname{diag}(\bar{S}(t)))^{-1}\{\operatorname{diag}(\bar{S}(t)) \sigma(t, C(t)) \theta(t, A(t)) \\
& \left.-E\left\{\operatorname{diag}(\bar{S}(t)) \sigma(t, C(t)) \theta(t, A(t)) \mid \mathscr{F}^{k}(t)\right]\right\} d t+d w(t) \\
& =\left[\theta(t, A(t))-E\left\{\theta(t, A(t)) \mid \mathscr{F}^{k}(t)\right\}\right] d t+d w(t) \\
& =[\theta(t, A(t))-\tilde{\theta}(t, \tilde{D}(t))] d t+d w(t)
\end{aligned}
$$

It follows that for any $\pi \in \mathscr{H}^{k}(v ; T)$

$$
\begin{aligned}
& E\left\{U\left(\bar{V}^{v, \pi}(T)\right)\right\} \\
& =E\left\{U\left(\exp \left[v+\int_{0}^{T} \pi^{\prime}(t) \sigma(t, C(t))\left[\theta(t, A(t)) d t+d w(t)-\frac{1}{2} \sigma^{\prime}(t, C(t)) \pi(t) d t\right]\right]\right)\right\} \\
& =E\left\{U\left(\exp \left[v+\int_{0}^{T} \pi^{\prime}(t) \sigma(t, C(t))\left[\tilde{\theta}(t, \tilde{D}(t)) d t+d \tilde{w}(t)-\frac{1}{2} \sigma^{\prime}(t, C(t)) \pi(t) d t\right]\right]\right)\right\} \\
& =E\left\{U\left(\bar{V}^{v, \pi, k}(T)\right)\right\}
\end{aligned}
$$

\subsection{Pricing according to the "Fernholz-Karatzas Approach"}

As we had seen in the complete observation case, in this approach the price corresponds to the minimal superreplicating initial capital and thus involves implicitly hedging strategies that are $\mathscr{F}^{k}$-adapted. In line with subsection 5.2.4 we then use the same methodology as in subsection 4.1 for the complete observation case, but applied here to the complete observation model that corresponds to the original incomplete observation model and was derived in the previous subsection 5.2.

When trying to carry over Theorem 3 to the present situation we face the problem that, although in addition to the primary assets also the empirical log-covariances are observable, the investment instruments are only the primary assets. We present now two possible variants of extensions of Theorem 3 .

\subsubsection{First Variant}

In this variant we completely ignore the empirical covariances and consider $\bar{S}(t)$ as the only observables, namely $C(t)$ is the $n$-dimensional vector given by $C(t)=\bar{S}(t)$ and satisfying the subsystem of (46) consisting of the first $n$ rows. The filtration $\mathscr{F}^{k}$ 
remains as defined in (47) and the filter has the same form as in (48) and we also keep Assumption 12. Furthermore, analogously to (49) we now put

$$
\tilde{D}(t)=\left(\bar{S}_{1}(t), \cdots, \bar{S}_{n}(t), \xi_{1}(t), \cdots, \xi_{q}(t)\right)^{\prime} .
$$

It is also easily seen that in the given situation $\mathscr{F}^{k}(t)=\mathscr{F}^{\tilde{w}}(t)$ with $\tilde{w}(t)$ as in (54). Replacing then $\theta(t)$ in (22) by $\tilde{\theta}(t, \tilde{D}(t))$ from (58), and using, instead of $Z(t)$ in (18), the process

$$
Z^{k}(t):=\exp \left\{-\int_{0}^{t} \tilde{\theta}^{\prime}(s, \tilde{D}(s)) d \tilde{w}(s)-\frac{1}{2} \int_{0}^{t}\|\tilde{\theta}(s, \tilde{D}(s))\|^{2} d s\right\}, 0 \leq t<\infty
$$

as "Radon-Nikodym process", it can be easily verified that the following extension of Theorem 3 holds

Theorem 20 Consider a given a maturity $T$ and a claim $Y^{k} \in \mathscr{F}^{k}(T)$ such that $E\left\{Y^{k} \frac{Z^{k}(T)}{B(T)}\right\} \in(0, \infty)$ with $Z^{k}(t)$ as in (66). For $t \in[0, T]$ and $s \in[t, T]$ denote by $V^{v, \pi, k}(s)$ the value in $s \geq t$ of a portfolio starting from $V^{v, \pi, k}(t)=v$ with $\pi(\cdot) \in$ $\mathscr{F}^{k}(\cdot)$. Let

$\mathscr{H}_{t}^{k}(v ; T):=\left\{\pi(\cdot) \in \mathscr{F}^{k}(\cdot) \mid P\left\{V^{v, \pi, k}(s) \geq 0, \forall t \leq s \leq T\right\}=1 ; V^{v, \pi, k}(t)=v \in \mathscr{F}^{k}(t)\right\}$

namely the set of admissible strategies starting from a capital $v$ at time $t$ and put

$$
\mathscr{U}^{Y^{k}}(t):=\operatorname{essinf}\left\{v>0 \mid \exists \pi \in \mathscr{H}_{t}^{k}(v ; T) \text { s.t. } V^{v, \pi, k}(T) \geq Y^{k}\right\}
$$

which represents the minimal hedging price at time t. Let, furthermore,

$$
Y^{k}(t):=\frac{B(t)}{Z^{k}(t)} E\left\{Y^{k} \frac{Z^{k}(T)}{B(T)} \mid \mathscr{F}^{k}(t)\right\}
$$

with $Z^{k}(t)$ as in (66) and with the expectation under the physical measure P. If $\mathscr{F}^{k}(t)=\mathscr{F}^{\tilde{w}}(t)$, where $\tilde{w}(t)$ is the $n$-dimensional Wiener process according to Remark 16, then

$$
Y^{k}(t)=\mathscr{U}^{Y^{k}}(t) .
$$

Proof. The proof follows step by step that of Theorem 3 (with the appropriate adaptation of symbols) whereby $M(s)$ in (23) is replaced by (recall that $\mathscr{F}^{k}(t)=\mathscr{F}^{\tilde{w}}(t)$ )

$$
M(s):=E\left\{Y^{k} \frac{Z^{k}(T)}{B(T)} \mid \mathscr{F}^{k}(s)\right\}=Y^{k}(t) \frac{Z^{k}(t)}{B(t)}+\int_{t}^{s} \psi^{\prime}(u) d \tilde{w}(u)
$$

and $\pi(s)$ turns out to be

$$
\pi(s)=\sigma^{-1}(s, C(s))\left(\frac{\psi(s)}{M(s)}+\tilde{\theta}(s, \tilde{D}(s))\right)
$$


for $s \in[t, T]$ with $t \in[0, T]$.

\subsubsection{Second Variant}

This is a more theoretical variant, where one imagines the possibility of investing also in the log-covariances (in general in all quantities that are supposed to be observable). In this case $C(t)$ and $\tilde{D}(t)$ remain as in section 5.2 where $C(t)$ satisfies (46) with $W(t)$ as in (45) (recall also that this implies that the $\operatorname{diag}(\bar{S}(t)) \sigma(t, C(t)$ ) of the first variant now becomes $G(t, C(t))$ ). Furthermore (see Remark 15), this time $\mathscr{F}^{k}(t)=\mathscr{F}^{\tilde{W}}(t)$ with $\tilde{W}(t)$ as in $(53)$. The extension of Theorem 3 for the present variant now reads exactly as Theorem 20 . In the proof of the Theorem for this second variant the changes with respect to the first variant concern, besides $C(t)$, the process $\tilde{\theta}(t, \tilde{D}(t))$ that now has to be of dimension $k=2 n$. The other changes concern $Z^{k}(t)$ in (66) that now becomes

$$
Z^{k}(t):=\exp \left\{-\int_{0}^{t} \tilde{\theta}^{\prime}(s, \tilde{D}(s)) d \tilde{W}(s)-\frac{1}{2} \int_{0}^{t}\|\tilde{\boldsymbol{\theta}}(s, \tilde{D}(s))\|^{2} d s\right\}, 0 \leq t<\infty
$$

with $\tilde{W}(t)$ as in (53). Furthermore, $M(t)$ here becomes

$$
M(s):=E\left\{Y^{k} \frac{Z^{k}(T)}{B(T)} \mid \mathscr{F}^{k}(s)\right\}=Y^{k}(t) \frac{Z^{k}(t)}{B(t)}+\int_{t}^{s} \psi^{\prime}(u) d \tilde{W}(u)
$$

with $\psi(t)$ a $2 n$-dimensional process and the hedging strategy is

$$
\pi(t):=\left(G^{-1}(t, C(t))\right)^{\prime}\left[\frac{\psi(t)}{M(t)}+\tilde{\theta}(t, \tilde{D}(t))\right]
$$

\subsection{Pricing According Platen's “Benchmark Approach"}

As we had seen in the complete observation case, Platen's approach is of the type of utility indifference pricing. In line with subsection 5.2.4 we then use the same methodology as in subsection 4.2 for the complete observation case, but applied here to the complete observation model that corresponds to the original incomplete observation model and was derived in subsection 5.2. Contrary to what happened for the Fernholz-Karatzas approach in subsection 5.3, where we had to consider two somewhat restricted variants, here it can be seen that we can follow the exact same steps as in the proof of Theorem 8 for the complete observation case (with the appropriate adaptation of symbols). We thus conclude that the following formula holds for the price $P^{k}(t)$ under incomplete observation with information level/observation dimension $k$

$$
P^{k}(t)=V^{\rho, k}(t) E\left\{\frac{Y^{k}}{V^{\rho, k}(T)} \mid \mathscr{F}^{k}(t)\right\}
$$


where the discounted version of $V^{\rho, k}(t)$ satisfies (63).

\subsection{Comments on the Equivalence of the Two Approaches in the Case of Incomplete Observations}

In section 4.3 we have shown that, in case of complete observations, the FernholzKaratzas (F.-K.) and Platen's benchmark (PB) approach lead to the same derivative prices. In the case of incomplete observations the formulas that we have derived in subsections 5.3 and 5.4 correspond to those in subsections 4.1 and 4.2 respectively and so the equivalence should also hold for incomplete observations. However, while PB can be applied also if not all observables can be used as investment instruments (in the case of this paper the empirical covariances), FK had to be restricted to situations where all observables are also investment instruments. This corresponds to intuition if one considers that FK relies on completing the market, while PB is of the utility indifference pricing type that is typically designed for incomplete markets. This difference did not come up in the complete observation case because there all investment instruments are automatically observable.

Acknowledgements Part of the contribution by the second author was obtained while he was visiting professor 2009 for the chair Quantitative Finance and Insurance at the LMU University in Munich funded by LMU Excellent. Hospitality and financial support are gratefully acknowledged as are the useful suggestions by the participants to a series of seminars on the topic of the paper.

\section{References}

1. Fernholz E.R., Stochastic Portfolio Theory, Springer Verlag, New York 2002.

2. Fernholz E.R., and I.Karatzas, Stochastic Portfolio Theory: an Overview, In: Handbook of Numerical Analysis; Volume XV "Mathematical Modeling and Numerical Methods in Finance" (A.Bensoussan and Q.Zhang eds.), North Holland, 2008, pp. 89-168.

3. Galesso G., Arbitraggio e diversificazione in informazione completa e parziale, Thesis, Univesity of Padova, 2008.

4. Liptser R., and A. Shiryaev, Statistics of Random Processes: I. General Theory, Springer Verlag 1977.

5. Platen E., and D.Heath, A Benchmark Approach to Filtering in Finance, Springer Finance, Berlin-Heidelberg 2006.

6. Platen E., and W.J. Runggaldier, A Benchmark Approach to Filtering in Finance, Asia-Pacific Financial Markets 11 (2005), pp. 79-105.

7. Platen E., and W.J. Runggaldier, A Benchmark Approach to Portfolio Optimization under Partial Information, Asia-Pacific Financial Markets 14 (2007), pp. 25-43.

8. Schachermayer W., Utility Maximization in Incomplete Markets. In: Stochastic Methods in Finance (M.Frittelli and W.Runggaldier, eds.) Lecture Notes in Mathematics Vol. 1856. Springer-Verlag, Berlin-Heidelberg, 2004, pp. 255-293. 